\title{
Suppressing intrinsic spin harmonics at the $\mathrm{AGS}^{1}$
}

\author{
A. Lehrach, E.D. Courant, J.W. Glenn, H. Huang, T. Roser
}

Brookhaven National Laboratory, P.O. Box 5000, Upton, New York 11973, USA

\section{Introduction}

An rf dipole is used in the AGS to preserve the polarization during crossing strong intrinsic resonances by exciting adiabatic spin flips [1]. Significant polarization losses have been observed at weak intrinsic resonances [2]. One method to conserve polarization at weak intrinsic resonances is a rapid change of the betatron tune at the resonance. Due to the non-adiabatic nature of this tune jump, the beam emittance is increased [3]. More convenient is a method called suppressing intrinsic spin harmonics, already successfully used at the Cooler Synchrotron COSY [4]. In this paper we describe how to use this method at the Alternating Gradient Synchrotron AGS.

\section{Depolarizing Resonances}

For an ideal planar circular accelerator with a vertical guide field, the particle spin vector precesses around the vertical axis. Thus the vertical beam polarization is preserved. The number of spin precessions per revolution of the beam in the ring is given by [5]

$$
\nu_{s p}=\gamma G
$$

where $G=1.7928$ is the gyromagnetic anomaly of the proton magnetic moment and $\gamma$ is the Lorentz factor. During acceleration of a polarized beam, depolarizing resonances are crossed if the precession frequency $\gamma G$ of the spin equals the frequency of the encountered spin-perturbing magnetic fields. Imperfection resonances are caused by magnetic field errors and misalignments of the magnets and intrinsic resonances are excited by horizontal fields due to the vertical focusing.

\section{Intrinsic Resonances in the AGS}

The number of intrinsic resonances depends on the superperiodicity $P$ given by the number of identical periods in the accelerator. The resonance condition for intrinsic resonances is given by

$$
\gamma G=k P \pm \nu_{y},
$$

\footnotetext{
${ }^{1}$ Work performed under the auspices of the U.S. Department of Energy
} 
where $k$ is an integer and $\nu_{y}$ is the vertical betatron tune. The strength of depolarizing intrinsic resonances is determinated by the structure of the unit cells in a circular accelerator. The AGS has a so called combined function lattice, where the beam is focused in the horizontally and vertically focusing gradients of the bending dipoles. It consists of 12 unit cells with one vertically focusing and one horizontally focusing quadrupole in each unit cell to adjust the betatron tunes. The magnetic structure of an individual unit cell is shown in Table 1 . The

\begin{tabular}{|c|c|c|c|c||c|c|c|c|c||c|c|c|c|c||c|c|c|c|c|}
\hline 1 & 2 & 3 & 4 & 5 & 6 & 7 & 8 & 9 & 10 & 11 & 12 & 13 & 14 & 15 & 16 & 17 & 18 & 19 & 20 \\
\hline $\mathrm{h}$ & $\mathrm{h}$ & $\mathrm{v}$ & $\mathrm{v}$ & $\mathrm{h}$ & $\mathrm{h}$ & $\mathrm{v}$ & $\mathrm{v}$ & $\mathrm{h}$ & $\mathrm{h}$ & $\mathrm{v}$ & $\mathrm{v}$ & $\mathrm{h}$ & $\mathrm{h}$ & $\mathrm{v}$ & $\mathrm{v}$ & $\mathrm{h}$ & $\mathrm{h}$ & $\mathrm{v}$ & $\mathrm{v}$ \\
\hline $\mathrm{s}$ & $\mathrm{s}$ & $\mathrm{l}$ & $\mathrm{l}$ & $\mathrm{l}$ & $\mathrm{l}$ & $\mathrm{l}$ & $\mathrm{l}$ & $\mathrm{s}$ & $\mathrm{s}$ & $\mathrm{s}$ & $\mathrm{s}$ & $\mathrm{l}$ & $\mathrm{l}$ & $\mathrm{l}$ & $\mathrm{l}$ & $\mathrm{l}$ & $\mathrm{l}$ & $\mathrm{s}$ & $\mathrm{s}$ \\
\hline
\end{tabular}

Table 1: Magnetic structure of the unit cell in the AGS. The AGS is composed of 12 unit cells, each unit cell consists of 20 long $(l)$ and short $(s)$ dipoles with horizontally $(h)$ and vertically $(v)$ focusing gradients. The two tuning quadrupoles located at position 3 and 17 an each unit cell. Symmetry points of this unit cell structure are at position 5 and 15 .

corresponding intrinsic resonances for superperiodicity $P=12$ in the momentum range of the AGS are summerized in Table 2. The intrinsic resonances $\gamma G=0+\nu_{y}, \gamma G=36-\nu_{y}$,

\begin{tabular}{|c|c||c|c|}
\hline $\mathrm{P}(\mathrm{GeV} / \mathrm{c})$ & $\gamma G$ & $\epsilon_{K}$ & $P_{f} / P_{i}$ \\
\hline \hline 4.5 & $0+\nu_{y}$ & $6.1 \cdot 10^{-3}$ & -0.42 \\
\hline 8.0 & $24-\nu_{y}$ & $0.2 \cdot 10^{-3}$ & 0.995 \\
\hline 10.8 & $12+\nu_{y}$ & $2.4 \cdot 10^{-3}$ & 0.45 \\
\hline 14.3 & $36-\nu_{y}$ & $5.1 \cdot 10^{-3}$ & -0.26 \\
\hline 17.1 & $24+\nu_{y}$ & $0.4 \cdot 10^{-3}$ & 0.98 \\
\hline 20.5 & $48-\nu_{y}$ & $0.6 \cdot 10^{-3}$ & 0.95 \\
\hline 23.4 & $36+\nu_{y}$ & $1.1 \cdot 10^{-3}$ & -0.77 \\
\hline 26.8 & $60-\nu_{y}$ & $6.4 \cdot 10^{-2}$ & -0.993 \\
\hline
\end{tabular}

Table 2: Resonance strengths $\epsilon_{K}$ of intrinsic resonances for a normalized emittance of $10 \pi \mathrm{mm}$ mrad. The ratio of preserved polarization at each resonance $P_{f} / P_{i}$ is calculated for a beam with $95 \%$ of the particles within a gaussian distribution with normalized emittance of $10 \pi \mathrm{mm}$ $\operatorname{mrad}$ and an acceleration rate of $\alpha=\frac{d \gamma G}{d \theta}=4.8 \cdot 10^{-5}$.

$\gamma G=36+\nu_{y}$, and $\gamma G=60-\nu_{y}$ are strong enough to use the existing $18 \mathrm{Gm}$ rf dipole. Some polarization losses have been observed at $\gamma G=12+\nu_{y}$. At the three weak intrinsic resonances $\gamma G=24-\nu_{y}, \gamma G=24+\nu_{y}$, and $\gamma G=48-\nu_{y}$ big polarization losses occur. The strength of this resonances is to weak to use the existing $\mathrm{rf}$ dipole.

\section{Optimizing the AGS lattice for a polarized beam}

The strength of the intrinsic resonances has been calculated by changing the strength of the existing quadrupoles at position 3 and 17. Fig. 1 shows the calculation for position 3 . In the betatron tune range between the vertical half integer betatron tune $\nu_{y}=8.5$ and the integer betatron tune $\nu_{y}=9.0$ the resonance strength of the three weak intrinsic resonances cannot be 


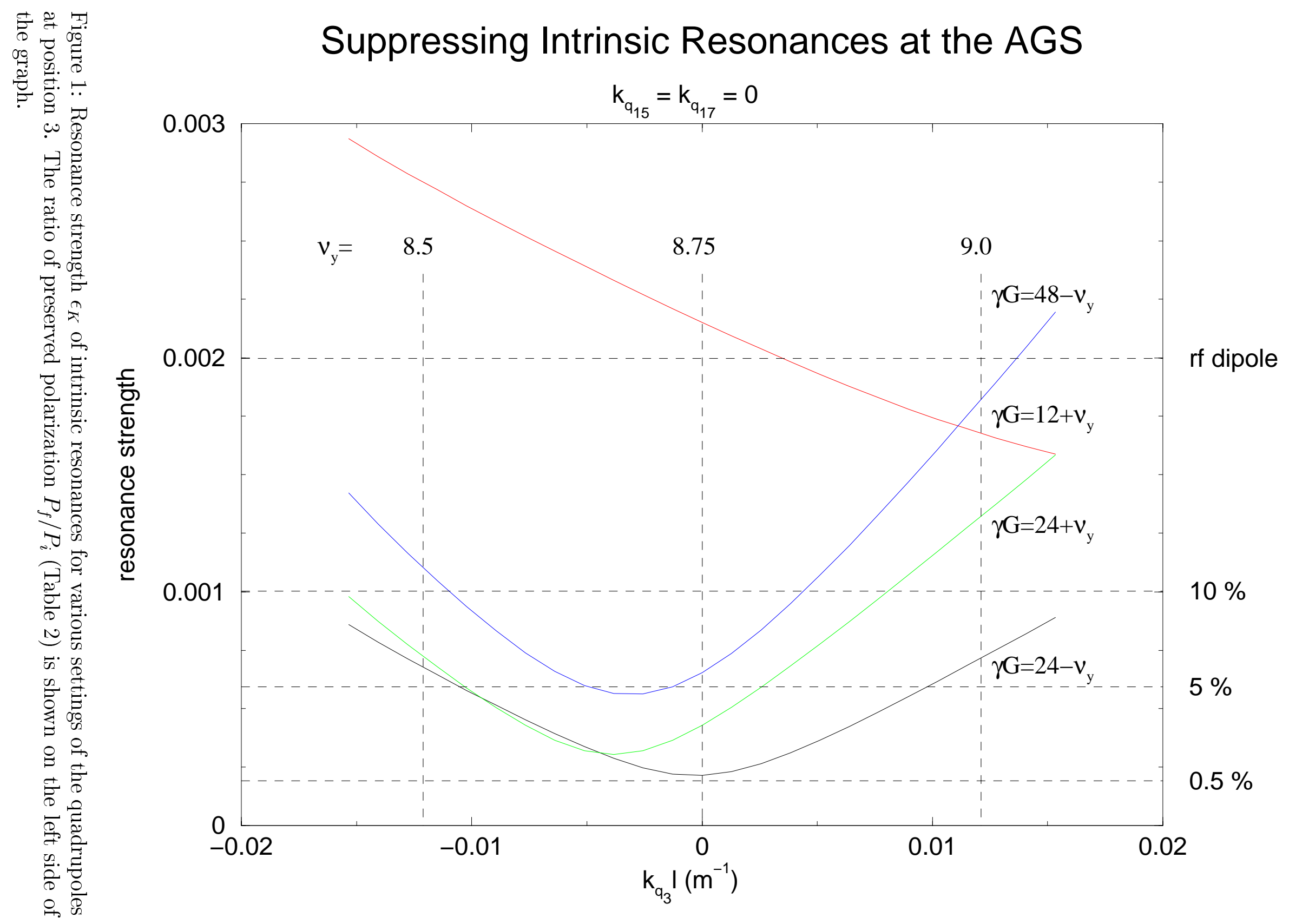




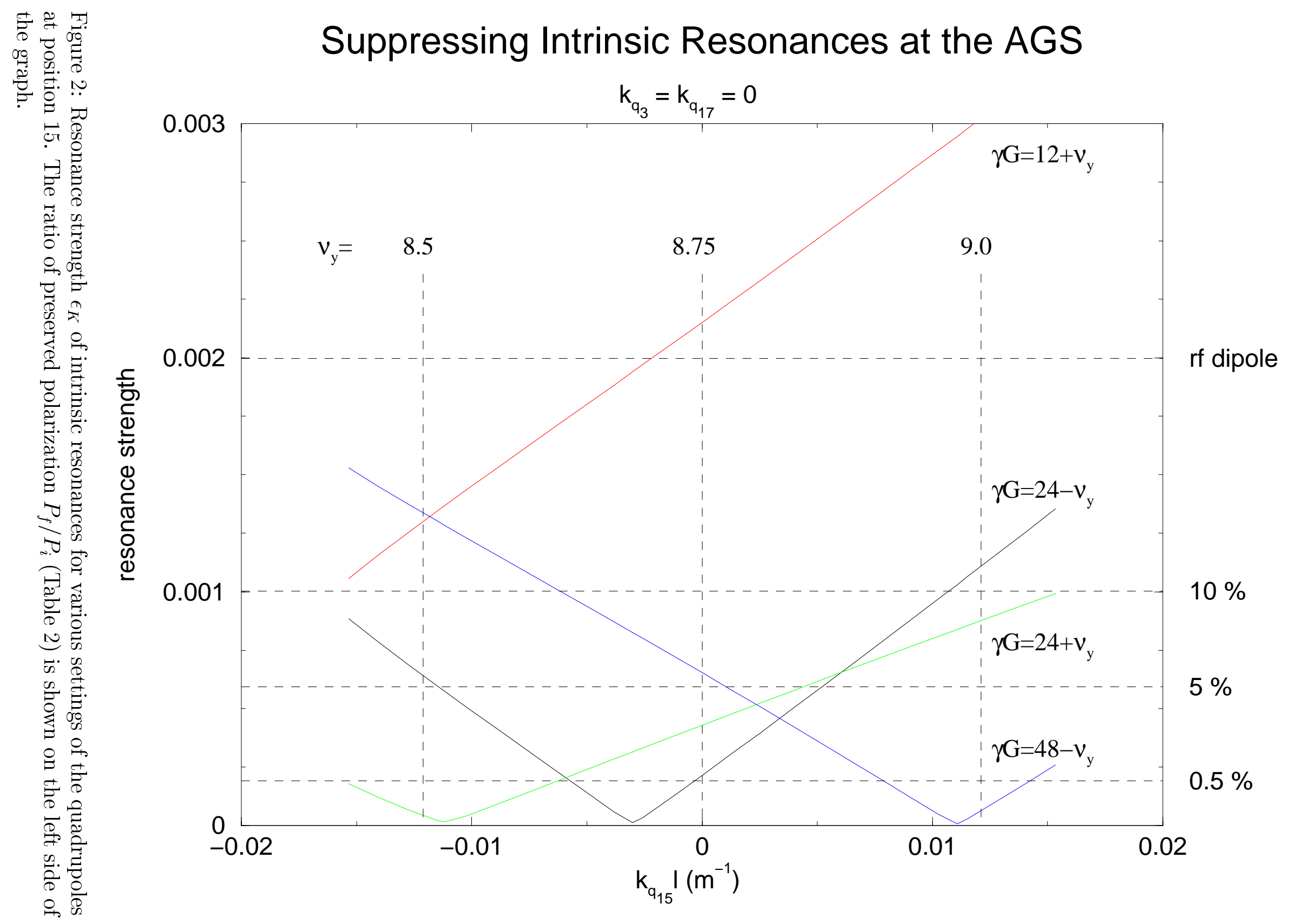


reduce to a strength with polarization losses less than $1 \%$. It is also not possible to excite the weak resonances strong enough to use the existing rf dipole. Basically the same behavior can be found if the strength of quadrupoles at position 17 is changed. In Fig. 2 the calculation for quadrupoles at position 15 are shown. It is possible to reduce the strength of the three weak intrinsic resonances to a polarization loss of less then $0.5 \%$. The betatron tunes are still between 8.55 and 8.95 , but cannot be controlled by the quadrupoles at position 3 and 17 . If one wants to control the betatron tune additional quadrupole have to be installed at position 5 , which is already occupied by extraction devices. It is also possible to use quadrupoles at the position 3 and 7 an the same time to control the betatron tune. Unfortunately position 7 is not available.

\section{Conclusion}

Theoretical studies of the AGS lattice revealed the possibility of reducing the strength of weak intrinsic resonances. Therefore, quadupoles have to be installed at position 15 in each unit cell. It can be expected that the method can be extended to the three weak intrinsic $\gamma G=24-\nu_{y}$, $\gamma G=24+\nu_{y}$, and $\gamma G=48+\nu_{y}$. The final polarization can be improved by an factor 1.14. It is also possible to increase the strength of the intermediate resonance $\gamma G=12+\nu_{y}$ in order to make an adiabatic spin flip using the rf dipole more efficient.

\section{References}

[1] M. Bai et al., Phys. Rev. E 56, 6002 (1997).

[2] T. Roser, Proceedings of the Workshop on Polarized Protons at High Energies, Hamburg DESY-PROC-1999-03 (1999).

[3] L.A. Ahrens, Operation of the AGS Polarized Beam, Proceedings 8th Symposium on High Energy Spin Physics, Minneapolis, AIP Conference Proceedings No. 187, 1068 (1988).

[4] A. Lehrach at al., 'Suppressing Intrinsic Spin Harmonics at the Cooler Synchrotron COSY', Nucl. Inst. Meth. A 429, 26 (2000).

[5] V. Bargman, L. Michel, V.L. Telegdi, Phys. Rev. Lett. 2, 435 (1959). 